\title{
Fast forward for systemic lupus erythematosus clinical trials
}

\author{
Mary K Crow
}

The 40-year drought since the FDA last approved a drug for systemic lupus erythematosus (SLE) is often cited to explain the sorely inadequate outcomes for patients with this disorder, and has provided a rationale for intensive research efforts. The length of the interval reflects the challenge in developing new therapies for this complex disease. By contrast, the relative ease of quantifying clinical responses in patients with rheumatoid arthritis (RA) and the availability of sufficient participants for clinical trials has facilitated the approval of new agents and effective use of drug combinations in this disease over the past 10 years. Multiple new biologic agents have been approved, beginning with etanercept, and have led to a dramatic reduction in RA-associated morbidity.

Among patients, rheumatologists, and foundations, advocacy for the development of new drugs for SLE is no less vigorous than for RA. The challenges facing the SLE research community have, however, been greater. The disease manifestations are diverse, there is no universally approved index against which to compare individual responses to therapy, and the number of patients who qualify for enrollment in clinical trials is limited by the lower prevalence of SLE compared with RA. Thus, the pharmaceutical companies have long shied away from initiating clinical trials of SLE therapy. However, the successful development of drugs for RA has, to some extent, stimulated interest in applying research advances in the disease mechanisms of SLE to drug development efforts.

Basic and translational research has identified molecules and cells that are attractive therapeutic targets in SLE. For instance, CD40 ligand, which is expressed at high levels on T cells in patients with SLE, was identified as a mediator of T-helper cell activity. Efforts to block its action with monoclonal antibodies were associated with complications, but therapeutic responses were encouraging (Grammer AC et al. [2003] J Clin Invest 112: 1506-1520). Studies of CD28 blockade to inhibit T-cell activation and
Basic and

translational

research has

identified

molecules

and cells that

are attractive

therapeutic

targets in SLE

MK Crow is the

Benjamin M Rosen

Chair in Immunology

and Inflammation

Research, Professor of

Medicine and Director

of Rheumatology

Research at the

Hospital for Special

Surgery, New York,

NY, USA.

Competing interests

MK Crow has declared associations with the

following companies/

organizations: Bristol-Myers

Squibb, Genentech, Idera

Pharmaceuticals, Novo

Nordisk and ZymoGenetics.

See the article online for full

details of the relationships.

www.nature.com/clinicalpractice doi:10.1038/ncprheum0866
T-helper cell activity are ongoing. Recognition of the important roles of B-lymphocyte stimulator (BLyS; also known as tumor necrosis factor ligand superfamily $13 \mathrm{~B}$ and B-cell activating factor of the tumor necrosis factor family) and interleukin 6 in B-cell survival and differentiation suggests that inhibition of the relevant pathways, or B-cell depletion, might be rational therapeutic approaches. The inhibition of interferon (IFN)- $\alpha$, which is present at elevated levels in many patients with SLE, is another compelling direction for drug development, as microarray studies have demonstrated elevated expression of IFN-inducible genes in patients with this disease (Crow MK et al. [2003] Autoimmunity 36: 481-490). Striking data from genome-wide association studies support these approaches: they identify gene variants associated with SLE that could potentially modulate T-lymphocyte and B-lymphocyte function, as well as the production of, and response to, IFN- $\alpha$ and other cytokines (International Consortium for Systemic Lupus Erythematosus Genetics (SLEGEN) et al. [2008] Nat Genet 40: 204-210).

Clinical trials testing at least 15 different therapeutic agents, many of them biologics that affect various pathways discussed above, are currently underway (http://clinicaltrials.gov/ct2/ results?term=lupus). This activity is an exciting demonstration of a meaningful change in the pace of drug development for SLE. Although SLE remains a highly heterogeneous disease, and the number of patients meeting enrollment criteria is a limiting factor for studies, efforts to validate biomarkers linked to relevant therapeutic targets might ultimately enable the design of clinical trials that select patients on the basis of their most relevant disease mechanism. A draft FDA guidance document outlining the paths to drug development for the treatment of SLE (http://www.fda.gov/cder/guidance/6496dft.pdf) and a spirit of cooperation among all constituencies have contributed to the current momentum toward the approval of novel drugs that could improve the health of SLE patients. 